\title{
Stress-Oriented Structural Optimization for Frame Structures
}

\author{
Shuangming Chai ${ }^{\mathrm{a}}$, Baiyu Chen ${ }^{\mathrm{a}}$, Mengyu Jia ${ }^{\mathrm{a}}$, Zhouwang Yang ${ }^{\mathrm{a}}$, Manfred Lau ${ }^{\mathrm{b}}$, Xiao-Ming Fu ${ }^{\mathrm{a}}$, Ligang Liu $^{\mathrm{a}, *}$ \\ ${ }^{a}$ School of Mathematical Sciences, University of Science and Technology of China, Hefei, China 230026 \\ ${ }^{b}$ School of Computing and Communications, Lancaster University, Lancaster, UK LA1 4WA
}

\begin{abstract}
To fabricate a virtual shape into the real world, the physical strength of the shape is an important consideration. We introduce a framework to consider both the strength and complexity of 3D frame structures. The key to the framework is a stress-oriented analysis and a semi-continuous condition in the shape representation that can both strengthen and simplify a structure at the same time. We formulate a novel semi-continuous optimization and present an elegant method to solve this optimization. We also extend our framework to general solid shapes by considering them as skeletal structures with non-uniform beams. We demonstrate our approach with applications such as topology simplification and structural strengthening.
\end{abstract}

Keywords: 3D printing, fabrication, stress analysis, optimization, topology simplification

\section{Introduction}

We have witnessed many developments in the area of computational fabrication in recent years [1, 2, 3, 4]. As 3D printers become increasingly common and affordable, there is a great need for tools that consider the physical properties of virtual objects. When bringing virtual objects into the real world through 3D printing, the strength of an object is one such important consideration. We present a framework to analyze both the strength and complexity of 3D frame structures, where a structure consists of a set of beams. The motivations for focusing on frame structures are that these structures are common in architectural models, they can also represent general 3D shapes, and they can be 3D printed as a real-world frame structure to represent the virtual shape without an excessive amount of printing material.

There has been some recent work exploring the idea of structural analysis of 3D printed shapes [5, 6, 7]. There also exists work that analyze structural parts and reduce the cost of $3 \mathrm{D}$ printing by building a skin-frame structure [8] or a honeycombcells structure [9]. However, previous methods optimize the structure problem iteratively between two parts: a geometry optimization and a topology optimization. In this paper, we introduce a single stress-oriented framework to analyze the strength and topology complexity of 3D frame structures simultaneously. A key contribution different from previous work is that we have a problem formulation and a semi-continuous condition in our shape representation. This condition can remove structurally redundant elements to reduce the overall shape complexity without sacrificing its structural strength.

\footnotetext{
${ }^{*}$ Corresponding author

Email addresses: kfckf ckf@mail.ustc.edu.cn (Shuangming Chai), chenby@mail .ustc.edu.cn (Baiyu Chen),

jimengyu@mail.ustc.edu.cn (Mengyu Ji), yangzw@ustc.edu.cn

(Zhouwang Yang), manfred. lau@gmail .com (Manfred Lau),

fuxm@ustc.edu.cn (Xiao-Ming Fu), lgliu@ustc. edu.cn (Ligang Liu)
}

We formulate our problem to optimize scalar parameters of a frame structure such that it is 3D printable with high strength, while taking into account the volume, semi-continuous, symmetric, and sparsity constraints. These constraints are quite intuitive, as they limit the size and complexity of the output structure while maintaining its aesthetics. The idea is to strengthen weak parts while maintaining the overall volume of the shape, and optionally changing the topology and keeping the shape symmetry. In particular, the semi-continuous constraint is key to our formulation, as it includes a choice between lower and upper bounds and a value of zero for each parameter in the shape representation. An element within a shape with a parameter value of zero will disappear. Our formulation of this condition allows us to explore the tradeoffs between strength and complexity in frame structures. The user can also control the tradeoff to choose among structures with various simplified topologies and high strength.

We use stress as a measure of strength of an object. We consider the frame structure as a set of beam elements and compute the stress of each element. Our stress-oriented structural optimization then minimizes the maximal stress of all elements. The semi-continuous condition in our shape representation requires a non-trivial solution to this problem. Hence we formulate a novel semi-continuous optimization and present an elegant method, the alternation direction method of multipliers (ADMM) algorithm, to solve it. Based on our framework on frame structures, we extend it to general tetrahedral meshes. By considering a tetrahedral mesh as a skeletal structure, the formulation and optimization method can be easily applied to a skeletal structure.

We demonstrate our framework over various 3D models of frame structures. We show the applications of the strengthening of weak parts and topology simplification while maintaining structural symmetry. Our results highlight the main concepts of the stress-oriented structural optimization. 
The contributions of our work are: (i) a stress-oriented framework to analyze both the strength and structural complexity of 3D frame structures with a semi-continuous condition; (ii) a semi-continuous optimization to minimize the maximal stress of a structure and an elegant method to solve this optimization; (iii) an extension to tetrahedral meshes by constructing skeletal structures and considering as frame structures; and (iv) applications of our framework to structural strengthening and topology simplification. Section 3 describes the stress analysis framework and the representation of an input shape as a frame structure. Section 4 describes our problem formulation including the objective function, the semi-continuous condition, and various constraints in our stress-oriented optimization. Section 5 describes a reformulation of the original problem formulation into a semi-continuous optimization such that it can be solved with the ADMM algorithm. Section 6 describes the extension of our framework to tetrahedral meshes.

\section{Related Work}

Structural Analysis for Fabrication. With the rapid development of techniques for 3D printing, many researchers have recently studied geometric processing problems for the purpose of fabrication. These fabrication-aware methods are typically led by a stress analysis that uses the finite element method. Stava et al. [5] introduce a method that analyzes the stress of a model and deforms it by hollowing, thickening and strut insertion. Zhou et al. [6] present a method to search for the worstcase stress under forces from all possible directions. Langlois et al. [10] use a stochastic finite element method to compute failure probabilities which can analyze the static soundness of one object. Zhou et al. [11] present a direct shape optimization method which penalizes geometric deviation while bounding the stress under specific external loads. Chen et al. [12] propose a finite element discretization scheme to use a reduced basis for fast stress analysis. Chen et al. [13] analyze elastic deformation caused by gravity to solve the inverse problem of computing a shape that when fabricated deforms naturally to a target shape. Prévost et al. [3] explore the problem of deforming shapes to make them physically balance. Among this area of work, we contribute a stress-oriented problem formulation for automatically strengthening a frame structure while simplifying its structural complexity.

Structural Simplification for Fabrication. There has been work on problems which aim to simplify the complexity of the structure of 3D shapes for fabrication. Many methods are based on decomposing a 3D shape into smaller pieces and then assembling them to form the original shape or a resemblance of it. Luo et al. [14] suggest a method to 3D print large objects by first segmenting an object into smaller parts and then assembling the parts to form the larger shape. Hildebrand et al. [15] create parts from a 3D shape that can then be fabricated and assembled in an optimal direction. Interlocked planar [16, 17] or solid pieces [18, 19, 20] can be used to form a shape that resembles the original. Zimmer et al. [21] approximate surfaces by building physical structures with the Zometool construction system. Vanek et al. [22] present a method to divide a mesh into parts which are then efficiently packed into space for $3 \mathrm{D}$ printing. Instead of decomposing a shape into smaller pieces, we simplify a frame structure by possibly removing elements from it. We contribute a semi-continuous optimization for this purpose.

Special Structure Design. Motivated by existing architectural structures, some types of special 3D printed structures have been explored. Some structures, like skin-frame structure [8] or honeycomb-cells structure [9], are designed to reduce the cost of $3 \mathrm{D}$ printing via stress analysis. These methods are used for constructing the interior supporting structure of a solid object and these structures are cost-effective and are stable with high strength. Some structures are designed as the support structure necessary for $3 \mathrm{D}$ printing. The reduction of support structure can save printing time and material [23, 4]. A bridge structure [4] can reduce the cost and meet stability conditions. Yang et al. [24] design a support-free structure to fabricate a balanced object without interior supports. Recently inspired by some materials in the nature, microstructures [25, 26, 27, 28] become popular, which are composed of tileable and printable small scale assemblies made of one or several materials. A framework is proposed [29] to generate statically sound and materially efficient frame structures with different types of cross sections. In this paper, we focus on strengthening and simplifying frame structures consisting of beams. We can also extend our framework to converting general meshes to skeletal representations for our analysis.

\section{Preliminaries}

This section describes the stress analysis and the representation of an input 3D shape of our algorithm as a frame structure. The stress computation described here is used in our optimization in Section 4

\subsection{Stress Computation}

In continuum mechanics, stress is a physical quantity that expresses the internal forces that neighboring particles of a continuous material exert on each other [30, 31]. The strength of a material is measured in force per unit area, which depends on its capacity to withstand axial stress, shear stress, bending, and torsion. A static elastic object satisfies the following equilibrium equation:

$$
\left\{\begin{aligned}
-\operatorname{div} \sigma(\mathbf{u})=\mathbf{f}, & & \text { in } \Omega, \\
\mathbf{u}=\mathbf{0}, & & \text { on } \Gamma_{H}, \\
\sigma(\mathbf{u}) \cdot \mathbf{n}=\mathbf{g}, & & \text { on } \Gamma_{N},
\end{aligned}\right.
$$

where $\mathbf{u}$ is the displacement, $\boldsymbol{\sigma}$ is the stress tensor, $\mathbf{f}$ is the body force, and $\mathbf{g}$ is the surface force. This differential equation is defined in the region of an object $\Omega, \Gamma_{H}$ and $\Gamma_{N}$ are two open subsets of the boundary of $\Omega$, such that $\partial \Omega=\bar{\Gamma}_{H} \cup \bar{\Gamma}_{N}$ and $\Gamma_{H} \cap \Gamma_{N}=\emptyset$. We take the discretized form of this system, i.e. the linear equilibrium equation:

$$
K \mathbf{u}=F,
$$




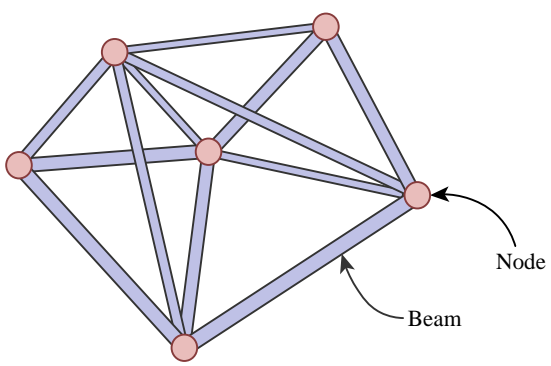

Figure 1: A frame structure consists of nodes and beams. The positions of nodes and the cross sections of beams defines the shape of a frame structure. For the sake of simplicity, we set each beam to be a cylindrical shape, which use a single radius to control the strength of a beam. The elastic properties of a frame structure are determined by its shape and the material of each beam.

where $K$ is the stiffness matrix and $F$ is the external loads including body forces and surface forces. Note that the displacement $\mathbf{u}$ is in the finite element space of piecewise linear continuous functions, which is different from the space in the continuous case (Equation 1).

\subsection{Frame Structure}

As shown in Figure 1, a frame structure consists of a set of beams and nodes where the beams are connected to each other at the nodes. In our framework, each beam is regarded as a simple cylindrical shape with a radius and a length. The beams defines the topology (i.e. the connectivity between nodes) of the frame structure. According to theory on frame structures [32, [33], the stiffness matrix $K$ in Equation (2) can be computed for a frame structure, where $K$ depends on the node positions and beam radii [8]. The forces in this equation are gravity or external loads we specify. We can then solve for the displacement and compute the stress for each beam in the frame structure. Although the stress varies in different parts of the beam, we only consider the largest stress for each beam.

\section{Problem and Formulation}

We describe our problem formally in this section, including the objective function in our optimization, various constraints, and the problem formulation. The stress analysis from Section 3 allows us to compute the stress in our optimization. The solution to the optimization is presented in Section 5 .

\subsection{Problem}

Our problem involves two components: stress strengthening and topology simplification.

Stress strengthening. Structural optimization aims to determine the best design according to some physical objectives (e.g. greatest strength, maximum rigidity) under some constraints. A structure fails the strength criterion when the stress (force divided by area of material) induced by the load is greater than the capacity of the structural material to resist the load without breaking, or when the strain (percentage extension) is so great that the element no longer fulfills its function. As acknowledged in the literature [34, 35, 36], it is natural to use stress or strain as criteria for the weakness measure of a target structure. Hence we use stress as a criterion for the purpose of structural strengthening.

Structural complexity. There exists many beams in our frame structures and some of these beams are not significant to the stress of the overall structure. We may therefore remove some of them without affecting the stress of the overall shape and to simplify its structural complexity.

Problem. The input to our problem is a frame structure of beams (shown as Figure 11. Considering the above two components, our problem is to perform simultaneous stress strengthening and structural complexity reduction of the input shape under some external loads. The output is a modified frame structure with a smaller number of beams while the stress of the shape is maintained or strengthened. The radii of some beams are adjusted while some beams are removed. The resulting shape may be $3 \mathrm{D}$ printed as a structure with high strength and simplified complexity.

\subsection{Objective Function}

Objective function. Let $\mathcal{M}$ be a mesh representing the frame structure. We adopt stress as a criterion for the weakeness measure of an object and minimize the maximal stress of the beams in the structure. The stress-oriented structural optimization problem can thus be formulated as:

$$
\mathbf{s}^{*}=\underset{\mathbf{s} \in \Theta}{\arg \min } \max _{\mathbf{p} \in \mathcal{M}(\mathbf{s})} \sigma(\mathbf{p}),
$$

where $\mathbf{S}$ is the vector of structural design variables, $\Theta$ is the collection of all feasible design variables, and $\sigma$ is the stress function. The solution of the above problem is $\mathbf{s}^{*}$, and $\mathcal{M}\left(\mathbf{s}^{*}\right)$ is then the output shape.

Design variables. The degree of freedom of the structural design space is large in general. Hence we simplify the problem by focusing on a piecewise scaling transformation for each element of the shape. Let $\mathcal{K}=\left(V_{\mathcal{K}}, E_{\mathcal{K}}\right)$ be the frame structure of a shape, where $V_{\mathcal{K}}$ and $E_{\mathcal{K}}$ represent the set of nodes and beams respectively. For every beam, we define a scalar $s_{i}, i \in\left\{1, \cdots,\left|E_{\mathcal{K}}\right|\right\}$ which is the scaling factor of the radius of each beam (See Figure 2). Let $\mathbf{s}=\left(s_{1}, s_{2}, \cdots, s_{\left|E_{\mathcal{K}}\right|}\right)$ be our set of structural design variables. We can perform many changes to the shape by adjusting these scalar variables. For example, we can adjust $s_{i}$ to strengthen weak parts. We can re-distribute volume in the overall shape by adjusting $s_{i}$ for multiple parts. If

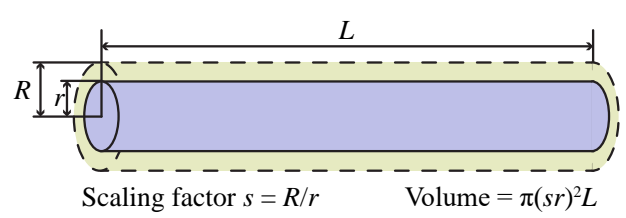

Figure 2: We choose our variables as the scaling factor for each beam. The scaling factor $s$ is defined by the ratio of radius and the volume can be computed by the corresponding scaling factor. A beam vanishes if we simply set $s=0$. 
we set $\mathbf{s}_{o}=\mathbf{1}=(1,1, \cdots, 1)$, then $\mathcal{M}\left(\mathbf{s}_{o}\right)$ is the original object. If we set some $s_{i}=0$ then the corresponding part will disappear and we can perform topological changes to the overall shape.

\subsection{Constraints}

We consider various constraints in our framework to maintain the size, aesthetics, and complexity of the output shape.

Volume constraint. We compute the shape's volume by adding the volume of all beams. Each beam's volume is estimated as the cross sectional area multiplied by its length. We can write the volume constraint as:

$$
\operatorname{Vol}(\mathbf{s}) \leq \gamma \operatorname{Vol}(\mathbf{1})
$$

where $\gamma$ is a user-specified value which means that the resulting volume is no more than $\gamma$ of the original volume.

Symmetry constraint. In many practical applications, it is important to maintain the symmetry of a shape during its structural optimization, so as to maintain its overall aesthetics. Our approach can achieve this by adding symmetry constraints as follows:

$$
s_{i}-s_{j}=0,(i, j) \in \mathcal{S},
$$

where $\mathcal{S}$ is the set of index pairs of beams that we wish to maintain symmetry with.

Semi-continuous constraint. In order to satisfy the printability of the object, the scalar $s_{i}$ should be within an interval $\left[a_{i}, b_{i}\right]$. For example, the scalar should be set such that the thickness of the shape is not less than the minimum manufacturable size of the $3 \mathrm{D}$ printer. Each scalar $s_{i}$ can also take a zero value. Hence this leads to a semi-continuous condition:

$$
s_{i} \in\left[a_{i}, b_{i}\right] \cup\{0\}, \quad i \in \mathcal{I}=\left\{1,2, \cdots,\left|E_{\mathcal{K}}\right|\right\} .
$$

Sparsity constraint. Moreover, we can add a cardinality constraint to control the overall complexity of the structure:

$$
\|\mathbf{S}\|_{0} \leq \tau
$$

\subsection{Formulation}

We can now formulate our stress-oriented structural optimization as follows:

$$
\begin{array}{cl}
\underset{\mathbf{s}}{\arg \min } & \max _{e \in \mathcal{E}} \sigma_{e}(\mathbf{s}) \\
\text { s.t. } & \operatorname{Vol}(\mathbf{s}) \leq \gamma \operatorname{Vol}(\mathbf{1}), \\
& s_{i}-s_{j}=0,(i, j) \in \mathcal{S}, \\
& s_{i} \in\left[a_{i}, b_{i}\right] \cup\{0\}, i \in \mathcal{I}, \\
& \|\mathbf{s}\|_{0} \leq \tau,
\end{array}
$$

where $\mathcal{E}$ is the set of beams in the frame structure, and $\sigma_{e}$ is the stress function described in the previous section.

\section{Semi-Continuous Optimization}

In this section, we present the method to solve the optimization described in Section 4 . In the formulation of our optimization (Equation (8)), the scaling factors $\mathbf{s}$ are a set of semicontinuous decision variables [37, 38]. Theoretically, it is in general NP-hard to solve this kind of highly nonlinear optimization problem which has combinatorial nature (semi-continuous sparsity) [39, 40]. Thus we reformulate our problem and then mathematically derive an algorithm based on the ADMM (alternating direction method of multipliers) strategy [41, 42] to solve it. The ADMM method is designed to solve convex optimization problems by breaking them into smaller pieces, and it has been applied to a number of problems arising in statistics and machine learning [43, 44]. The semi-continuous condition in our problem formulation leads to two sets of variables. The idea of ADMM is to split the optimization into sub-problems that iteratively find solutions to the two sets of variables.

\subsection{Problem Reformulation}

We add a new variable $\delta$ to represent the maximum stress and introduce a variable splitting strategy (and a new variable y) to reformulate the problem in Equation (8) as:

$$
\begin{aligned}
\underset{(\mathbf{s}, \delta, \mathbf{y})}{\arg \min } & \delta \\
\text { s.t. } & \sigma_{e}(\mathbf{s})-\delta \leq 0, e \in \mathcal{E}, \\
& \operatorname{Vol}(\mathbf{s}) \leq \gamma \operatorname{Vol}(\mathbf{1}), \\
& s_{i}-s_{j}=0,(i, j) \in \mathcal{S}, \\
& \mathbf{s}-\mathbf{y}=0, \\
& \mathbf{y} \in \mathbf{y},
\end{aligned}
$$

where $\mathcal{Y}=\left\{\mathbf{y} \mid a_{i} z_{i} \leq y_{i} \leq b_{i} z_{i}, z_{i} \in\{0,1\}, i \in \mathcal{I} ; \mathbf{1}^{T} \mathbf{z} \leq \tau\right\}$. The optimal $\mathbf{s}^{*}$ is our solution. The advantage of introducing the variable $\mathbf{y}$ is that it allows the decoupling of the continuous constraint and the semi-continuous sparsity constraint, since each of them now applies to one specific optimization variable $\mathbf{s}$ or $\mathbf{y}$. Although we introduce an additional constraint, it is now easier to solve the reformulated problem (Equation (9)) than the original one (Equation (8)).

\subsection{Solution}

Our solution is based on ADMM which is itself based on an augmented Lagrangian function and two sub-problems within the overall optimization. We describe these in detail in this subsection.

Augmented Lagrangian. One typical way for solving such an optimization problem is to use an augmented Lagrangian approach [45]. We use this method and define the problem's augmented Lagrangian function as follows:

$$
L_{\rho}(\mathbf{s}, \delta, \mathbf{y}, \lambda)=\delta+\lambda^{T}(\mathbf{s}-\mathbf{y})+\frac{\rho}{2}\|\mathbf{s}-\mathbf{y}\|^{2}
$$

with $\lambda$ being the Lagrangian multipliers and $\rho$ a positive parameter that balances the quadratic penalization. 


\section{$\overline{\text { Algorithm } 1 \text { The ADMM algorithm for structural optimization }}$}

Input: an initial structure $\mathcal{M}\left(\mathbf{s}^{o}\right)$

Step 0: Set $k=0$ and initialize $\mathbf{y}^{0}, \lambda^{0}$. Set the penalty parameter $\rho$ and the step-size $\alpha$;

\section{Repeat}

Step 1: Solve the (s, $\delta)$-subproblem

$$
\left(\mathbf{s}^{k+1}, \delta^{k+1}\right)=\underset{(\mathbf{s}, \delta) \in \mathcal{D}}{\arg \min } L_{\rho}\left(\mathbf{s}, \delta, \mathbf{y}^{k}, \lambda^{k}\right) ;
$$

Step 2: Solve the $\mathbf{y}$-subproblem

$$
\mathbf{y}^{k+1}=\underset{\mathbf{y} \in \mathcal{Y}}{\arg \min } L_{\rho}\left(\mathbf{s}^{k+1}, \delta^{k+1}, \mathbf{y}, \lambda^{k}\right) ;
$$

Step 3: Update the Lagrangian multipliers

$$
\lambda^{k+1}=\lambda^{k}+\alpha \rho\left(\mathbf{s}^{k+1}-\mathbf{y}^{k+1}\right) ;
$$

Until stopping criterion is met.

Output: an optimized frame structure $\mathcal{M}\left(\mathbf{s}^{*}\right)$ with its optimal variable $\mathbf{s}^{*}$.

Pseudocode. Instead of a joint optimization on the two variables, the idea of ADMM is to optimize alternatively over $(\mathbf{s}, \delta)$ and $\mathbf{y}$. Algorithm 1 gives the pseudocode of our solution with ADMM. The stopping criterion in the optimization is: the value of the function has almost no change or the number of iteration steps reaches a given upper bound.

$(\mathbf{s}, \delta)$-subproblem. The (s, $\delta)$-subproblem in ADMM involves a quadratic objective and the continuous constraint. We use the interior-point method to solve the subproblem:

$$
\min _{(\mathbf{s}, \delta) \in \mathcal{D}} \delta+\frac{\rho}{2}\left\|\mathbf{s}-\left(\mathbf{y}^{k}-\lambda^{k} / \rho\right)\right\|^{2},
$$

where $\mathcal{D}=\left\{(\mathbf{s}, \delta) \mid \sigma_{e}(\mathbf{s})-\delta \leq 0, e \in \mathcal{E} ; \operatorname{Vol}(\mathbf{s}) \leq \gamma \operatorname{Vol}(\mathbf{1}) ; s_{i}-\right.$ $\left.s_{j}=0,(i, j) \in \mathcal{S}\right\}$ is the feasible set of continuous variables.

$\mathbf{y}$-subproblem. We rewrite the $\mathbf{y}$-subproblem as a closed-form solution, starting with:

$$
\min _{\mathbf{y} \in \mathcal{Y}}\left\|\mathbf{y}-\left(\mathbf{s}^{k+1}+\lambda^{k} / \rho\right)\right\|^{2}=\sum_{i}\left(y_{i}-t_{i}\right)^{2}
$$

with $t_{i}=s_{i}^{k+1}+\lambda_{i}^{k} / \rho$. If $y_{i} \neq 0$, we denote

$$
\zeta_{i}=\min _{\mathbf{y} \in \mathcal{Y}}\left(y_{i}-t_{i}\right)^{2}= \begin{cases}\left(a_{i}-t_{i}\right)^{2}, & t_{i}<a_{i}, \\ 0, & a_{i} \leq t_{i} \leq b_{i}, \\ \left(b_{i}-t_{i}\right)^{2}, & t_{i}>b_{i} .\end{cases}
$$

Then this subproblem can be further simplified as:

$$
\begin{array}{ll}
\min _{\mathbf{z}} & \sum_{i} t_{i}^{2}\left(1-z_{i}\right)+\zeta_{i} z_{i}=\sum_{i} t_{i}^{2}-\sum_{i}\left(t_{i}^{2}-\zeta_{i}\right) z_{i} \\
\text { s.t. } & z_{i} \in\{0,1\}, i \in \mathcal{I} ; \mathbf{1}^{T} \mathbf{z} \leq \tau .
\end{array}
$$

Let $\left\{t_{\ell_{1}}^{2}-\zeta_{\ell_{1}}, t_{\ell_{2}}^{2}-\zeta_{\ell_{2}}, \cdots, t_{\ell_{\tau}}^{2}-\zeta_{\ell_{\tau}}\right\}$ be the first $\tau$ positive numbers of sequence $\left\{t_{i}^{2}-\zeta_{i}\right\}_{i \in \mathcal{I}}$ in descending order. The problem in

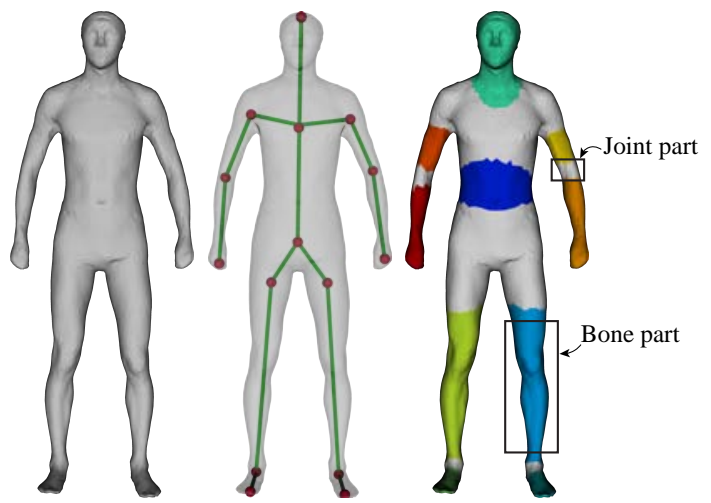

Figure 3: Converting a tetrahedral structure into a skeletal representation. Left: The input mesh; Middle: The skeleton of the mesh; Right: The mesh is decomposed into a set of bone parts (in colors) and joint parts (in gray).

Equation (13) has the solution $z_{\ell_{1}}=z_{\ell_{2}}=\cdots=z_{\ell_{\tau}}=1 ; z_{i}=$ $0, i \notin\left\{\ell_{1}, \ell_{2}, \cdots, \ell_{\tau}\right\}$. Finally, we get a closed-form solution

$$
y_{i}=\max \left(a_{i}, \min \left(t_{i}, b_{i}\right)\right) \cdot z_{i}, \quad \forall i \in \mathcal{I}
$$

for the $\mathbf{y}$-subproblem (Equation (12).

\section{Extensions}

We also extend our framework to skeletal shapes, which need to be converted into a tetrahedral structure for the finite element analysis. However, the number of tetrahedral elements in the overall structure may be large which can potentially lead to a large stiffness matrix and slow computation. Hence we simplify the computation by converting it into a skeletal representation (Figure 3), which can be considered as a frame structure of non-uniform beams.

Our skeletal representation and computation of stiffness matrices are similar to that of [7]. We first extract a skeleton from the input mesh. The mesh is then decomposed into a set of domains according to the bones of the skeleton, where each domain consists of its own set of vertices and tetrahedrons. Each tetrahedron belongs to only one domain. Some vertices may exist in multiple domains and these are duplicated. As shown in Figure 3, each domain is classified as either bone part (in colors) or joint part (in gray), where most parts of the mesh are bone parts. A bone part is associated with only one bone and

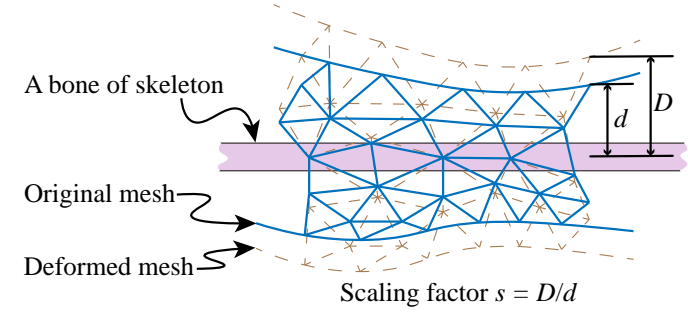

Figure 4: Our design variables are scaling factors related to every bone of the skeletal structure. All the tetrahedra belonging to a bone can scale at once according to the scaling factor. 


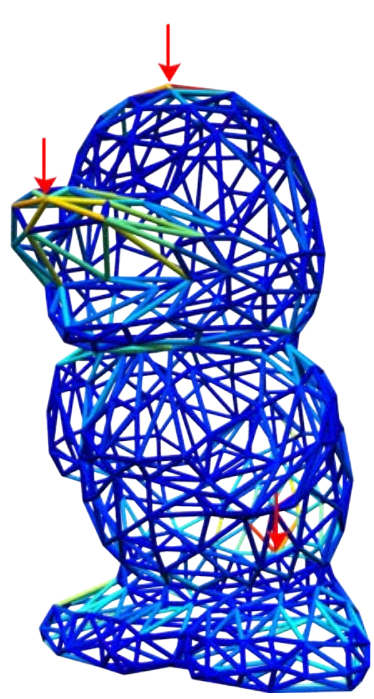

(a)

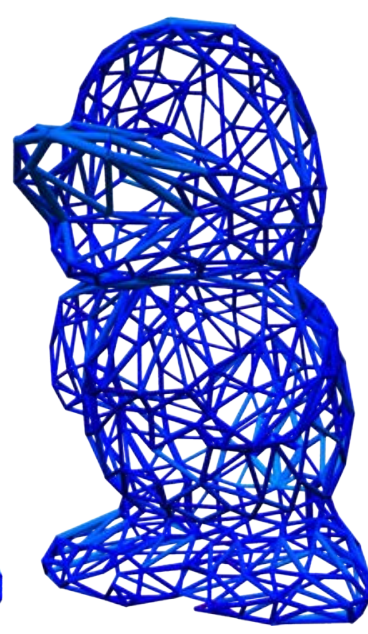

(b)

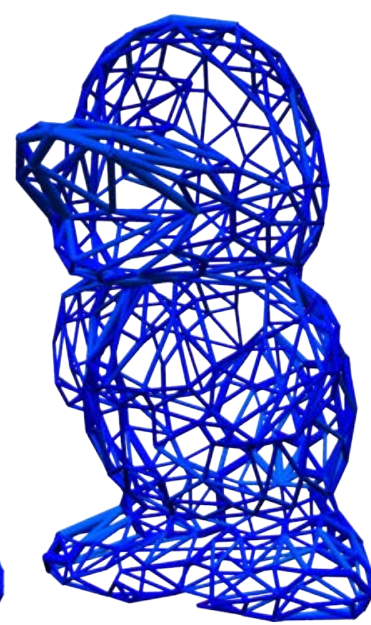

(c)

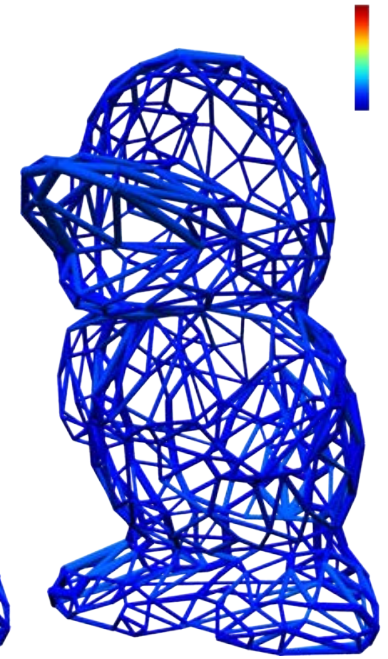

(d)

Figure 5: The results of a frame structure of a duck. The figures are colored according to the stress for each beam with red meaning large stress and blue meaning small stress. (a) The input duck model with 1107 beams. Three external loads (marked as red arrows) are applied on the model. (b, c, d) The simplified duck models (with 1007, 957, 907 beams respectively) obtained with our approach. The resulting models are increasingly simplified while their structures still remain strong.

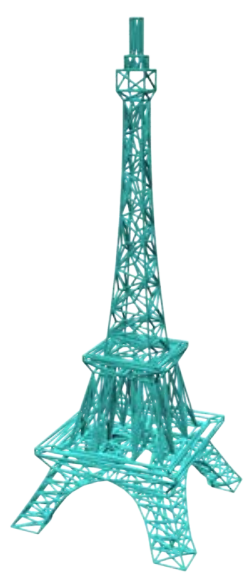

(a)

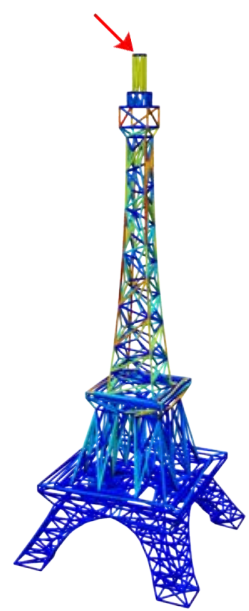

(b)

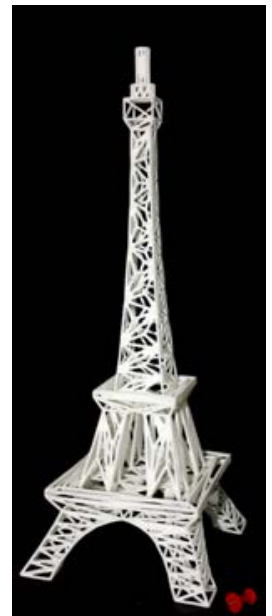

(c)

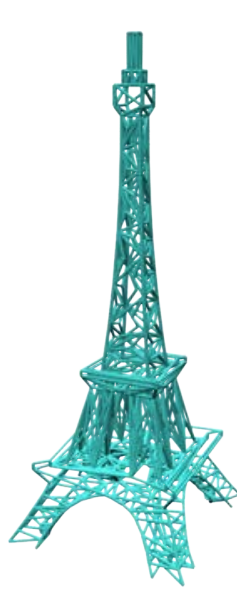

(d)

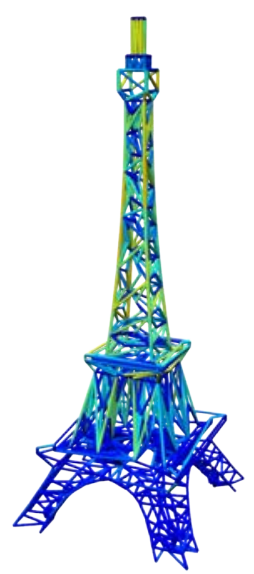

(e)

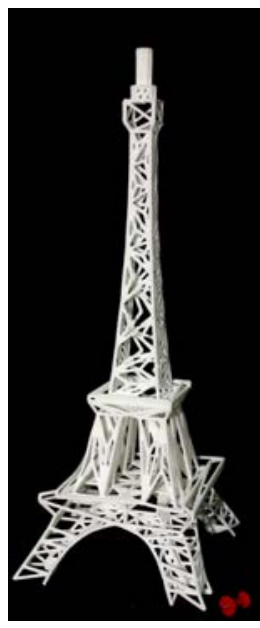

(f)

Figure 6: Results of the eiffel tower model. (a) The input frame model with 1521 beams. (b) The computed stress (colored with red being large and blue being small) of the input model under an external load of $2 \mathrm{~N}$ (shown by red arrow). (c) A photo of the SLS 3D printed model of the input. A small red pin is placed under the object as a size reference. (d) The simplified frame model with 1341 beams obtained by our approach. (e) The computed stress of the output model under the same external load as that of the input in (b). The output model has higher strength and reduced complexity. (f) The photo of the SLS 3D printed model of our output.

we can compute the stiffness matrix from the associated vertices and tetrahedra. A joint part is associated with multiple bones and the vertex coordinates associated with each bone are blended.

After the extrusion of the skeletal structure, we apply our framework to general meshes. The design variables are scaling factors, so the scaling factor is defined for every bone of the skeleton (See Figure 4). Then, the formulation and optimization are similar, except for the computation of stress, i.e. the use of von Mises stress.

A skeletal mesh shape is represented as a tetrahedral structure which consists of a set of vertices and a set of tetrahedral el- ements. For each tetrahedral element, we compute the element stiffness matrix and then assemble them into the total stiffness matrix $K$ in Equation (2) as in [5, 6]. To compute the strength of each element, we use the von Mises yield criterion and compute the von Mises stress.

\section{Experimental Results}

We have implemented our algorithm and tested it on various models. All the examples presented in this paper were created with a dual-core $3.5 \mathrm{GHz}$ machine with $8 \mathrm{G}$ memory. We have $3 \mathrm{D}$ printed some of the models to demonstrate our results. 


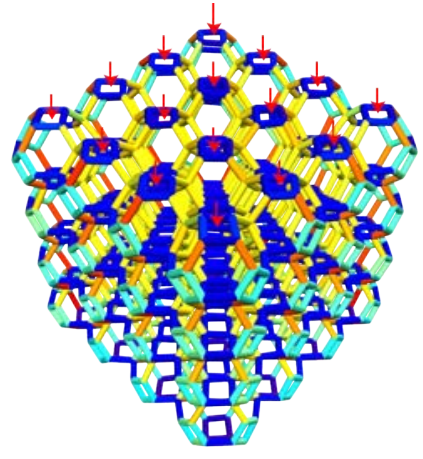

(a)

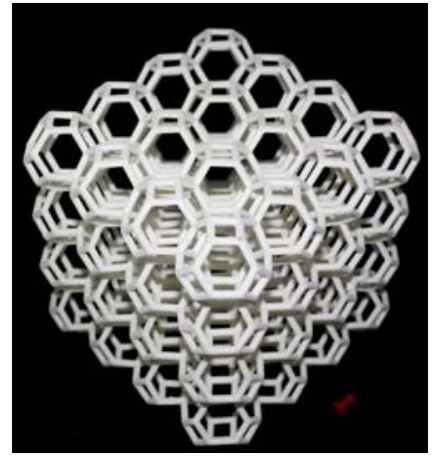

(b)

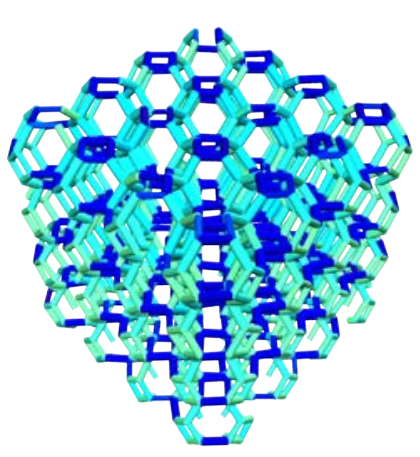

(c)

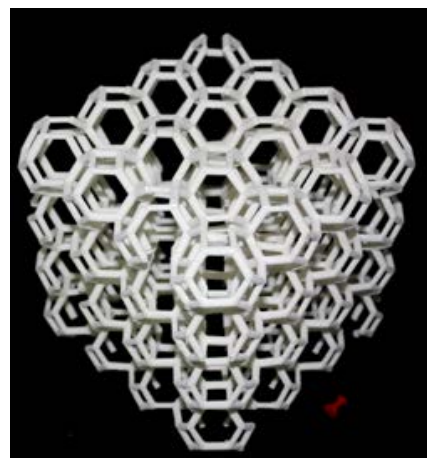

(d)

Figure 7: Results of the hardstruct model. (a) The input model where external loads are denoted as red arrows. The hardstruct model has 1728 beams. (b) We 3D printed the input model with a Sinterstation SLS 3D printer. A small red pin is placed under the object as a size reference. (c) The resulting model obtained by our approach is structurally simplified and strengthened with our approach. The hardstruct model has 1548 beams. (d) We physically demonstrate our results by $3 \mathrm{D}$ printing the output model.

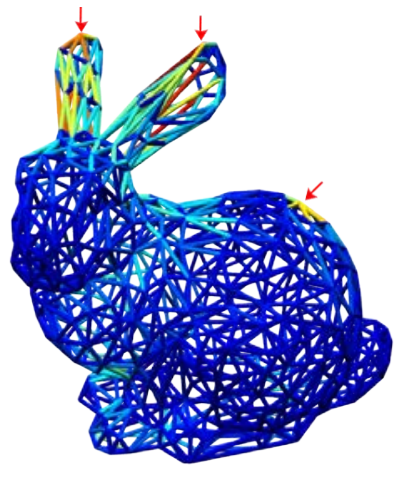

(a)

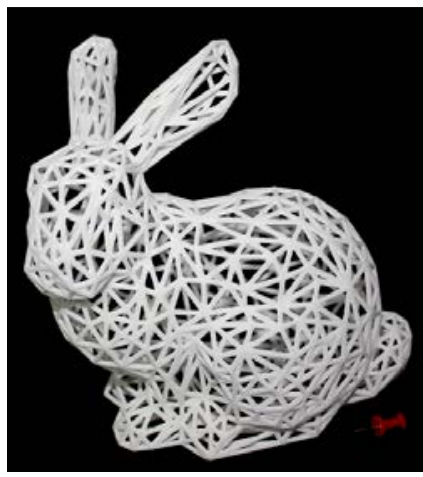

(b)

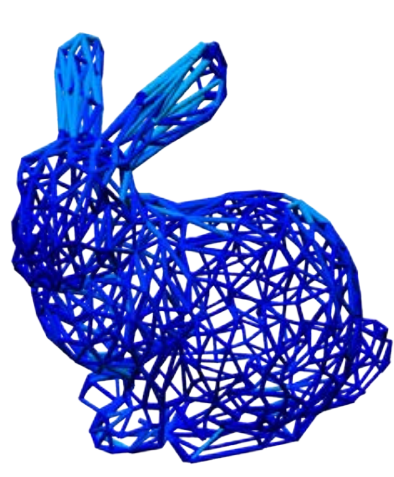

(c)

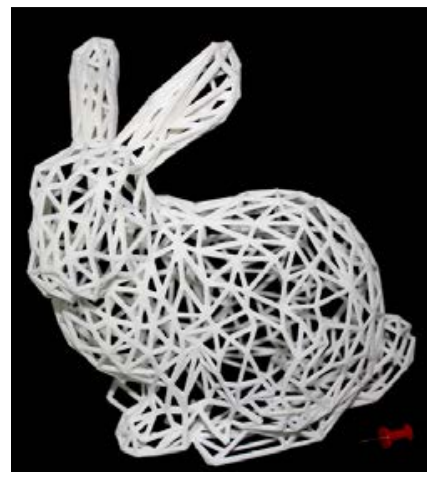

(d)

Figure 8: Our approach analyzes an input 3D frame structure to perform simultaneous structural strengthening and simplification for 3D printing. (a) The input bunny frame model with 1569 beams. The external loads are denoted as red arrows. (b) A photo of the SLS 3D printed models of the input. A small red drawing pin is placed under the object as a size reference. (c) The resulting model obtained by our approach. The number of beams is reduced to 1319 . (d) A photo of the SLS 3D printed models of our output.

Parameters. There are several parameters in our formulation. The user can change the value of the weight $\gamma$ to control the volume of the resulting object. We set $\gamma=1$ in our implementation which means that we do not want to increase the volume of the result adjusting the shape. Another parameter is $\tau$ which measures the complexity of the structure by measuring the number of beams. Thus $\tau$ can be adjusted by the user and set to a number less than the total number of beams in the input model. We specify the external loads manually at various points of the input shapes. In the optimization, we set the penalty parameter $\rho$ to be 10 and the step-size $\alpha$ to be 0.6 .

Figure 5 shows the results of our algorithm on a frame structure of a duck model. We set different values of the sparsity parameter $\tau$ and obtain a sequence of results. The resulting structural shapes in Figure 5 (b,c,d) are progressively simplified in the number of beams while their strengths are maintained.

Frame structures. We have tested our algorithm on various models of frame structures. Figure 6 shows an example of Eiffel tower. We give an external load as input and optimize the maximal stress. From the color in the figure, we can see that the maximal stress is lower than it before the optimization. Since the feet of the tower are fixed, the beams connecting fixed nodes have zero stress, thereby being removed. Figure 7 and Figure 8 show the results of a complicated 'hardstruct' structure and a bunny. For the 'hardstruct' model, we set the external forces to be evenly distributed downward forces on its top nodes. For the bunny model, we set three exteranl forces on three specified positions. These frame structures are structurally strengthened and topologically simplified while their total volumes are preserved.

Figure 9 shows two more examples whose external loads are vertical down. For the boomerang, since the given external force is vertical down, some horizontal beams have less contribution to the soundness, thus being removed by our optimization. The airport model has a constant gravity on each node. Note that there are also some horizontal beams removed due to the reason mentioned before.

Skeletal shapes. As an extension of our algorithm, we also test our algorithm on more general solid models, which are regarded as skeletal structures with non-uniform beams. By using our 

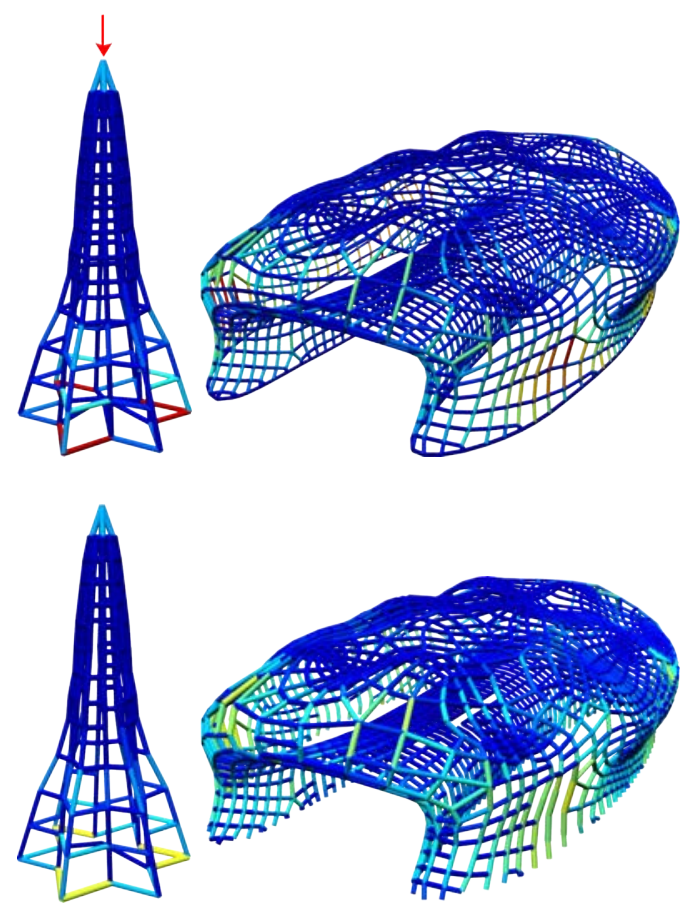

Figure 9: Results of applying our approach on the boomerang model (left) and the airport model (right). An external load (shown in red arrow) is applied on the boomerang model. Gravity is applied as external load to the airport model. The original models (top) have 312 and 5045 beams respectively. Our results (bottom) have 284 and 4585 beams respectively and they are both structurally simplified and strengthened compared to the originals.

method, the stress of the models can be automatically enhanced by thickening the weak parts of the models. We fabricated some of these models with FDM printers (see Figure 10.

\section{Conclusion}

We present a novel approach for performing structural analysis on 3D shapes with simultaneous structural strengthening and simplification. The optimized shape can be 3D printed with high strength and reduced complexity. We use stress as a criterion for measuring strength and minimize the maximal stress of the shape to formulate an optimization with a semicontinuous condition. We then derive an algorithm based on the ADMM method to solve the optimization and show its applicability and feasibility towards topology simplification and structural strengthening. Although this paper focuses on frame structures, our framework can be extended to general meshes by considering them as skeletal tetrahedral structures.

Our approach has some limitations. One limitation of our work is that we do not move the positions of the nodes in a structure. This means that although the structure can be much simplified, the inherent topology of the original structure remains the same. Moving the positions of the nodes can potentially be done in our optimization framework. However, this will enlarge the dimensions of the variable space and will need higher computational cost. We leave this as a future research direction.

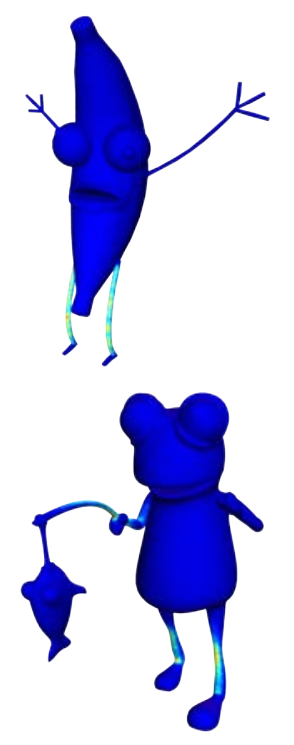

(a)

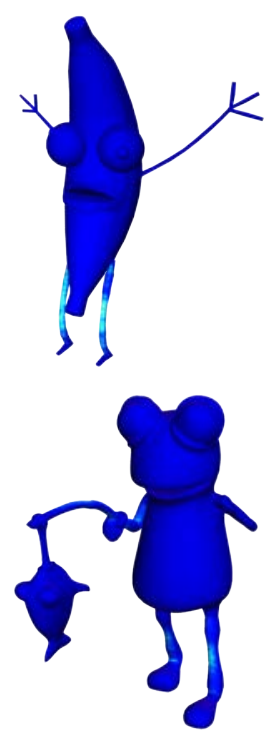

(b)

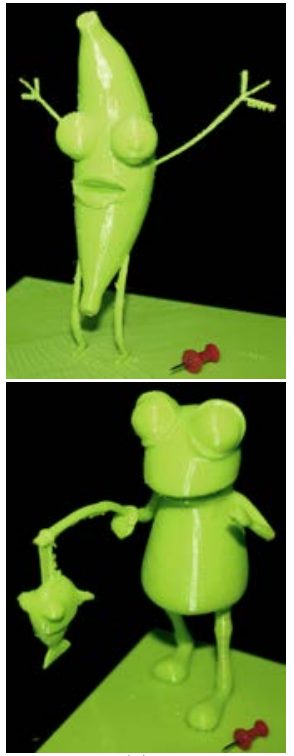

(c)
Figure 10: Examples of applying our approach on skeletal shapes (top: bananaman; bottom: fishing frog). (a) The original meshes; (b) Our results; (c) Photos of the FDM printed objects of our results. Note that the color shows the stress distribution

Another limitation is that the symmetry constraints may be difficult and/or tedious for humans to specify. In addition, as humans are able to easily identify any lack of symmetry, some of our simplified models may not be visually appealing as the topology may be simplified into non-intuitive patterns. We may consider this as an aesthetic constraint in the optimization in future work.

Another area of future work that is relevant for fabrication is the computation of supporting structures for FDM 3D printing. There has already been previous work in this area recently. Applying our stress-oriented structural optimization to this problem would be an interesting direction.

\section{References}

[1] B. Bickel, M. Bächer, M. A. Otaduy, H. R. Lee, H. Pfister, M. Gross, W. Matusik, Design and fabrication of materials with desired deformation behavior, ACM Transactions on Graphics (TOG) 29 (4) (2010) 63.

[2] S. Coros, B. Thomaszewski, G. Noris, S. Sueda, M. Forberg, R. W. Sumner, W. Matusik, B. Bickel, Computational design of mechanical characters, ACM Transactions on Graphics (TOG) 32 (4) (2013) 83.

[3] R. Prévost, E. Whiting, S. Lefebvre, O. Sorkine-Hornung, Make it stand: Balancing shapes for 3D fabrication, ACM Transactions on Graphics (TOG) 32 (4) (2013) 81.

[4] J. Dumas, J. Hergel, S. Lefebvre, Bridging the gap: Automated steady scaffoldings for 3D printing, ACM Transactions on Graphics (TOG) 33 (4) (2014) 98.

[5] O. Stava, J. Vanek, B. Benes, N. Carr, R. Měch, Stress Relief: Improving structural strength of 3D printable objects, ACM Transactions on Graphics (TOG) 31 (4) (2012) 48.

[6] Q. Zhou, J. Panetta, D. Zorin, Worst-case structural analysis, ACM Transactions on Graphics (TOG) 32 (4) (2013) 137.

[7] Y. Xie, W. Xu, Y. Yang, X. Guo, K. Zhou, Agile structural analysis for fabrication-aware shape editing, Computer Aided Geometric Design 35 (5) (2015) 163-179. 
[8] W. Wang, T. Y. Wang, Z. Yang, L. Liu, X. Tong, W. Tong, J. Deng, F. Chen, X. Liu, Cost-effective printing of 3D objects with skin-frame structures, ACM Transactions on Graphics (TOG) 32 (6) (2013) 177.

[9] L. Lu, A. Sharf, H. Zhao, Y. Wei, Q. Fan, X. Chen, Y. Savoye, C. Tu, D. Cohen-Or, B. Chen, Build-to-last: Strength to weight 3D printed objects, ACM Transactions on Graphics (TOG) 33 (4) (2014) 97.

[10] T. Langlois, A. Shamir, D. Dror, W. Matusik, D. I. Levin, Stochastic structural analysis for context-aware design and fabrication, ACM Transactions on Graphics (TOG) 35 (6) (2016) 226.

[11] Y. Zhou, E. Kalogerakis, R. Wang, I. R. Grosse, Direct shape optimization for strengthening 3d printable objects, Computer Graphics Forum 35 (7) (2016) 333-342.

[12] X. Chen, C. Zheng, K. Zhou, Example-based subspace stress analysis for interactive shape design, IEEE Transactions on Visualization and Computer Graphics 23 (10) (2017) 2314-2327.

[13] X. Chen, C. Zheng, W. Xu, K. Zhou, An asymptotic numerical method for inverse elastic shape design, ACM Transactions on Graphics (TOG) 33 (4) (2014) 95.

[14] L. Luo, I. Baran, S. Rusinkiewicz, W. Matusik, Chopper: Partitioning models into 3d-printable parts., ACM Transactions on Graphics (TOG) 31 (6) (2012) 129.

[15] K. Hildebrand, B. Bickel, M. Alexa, Orthogonal slicing for additive manufacturing, Computers \& Graphics 37 (6) (2013) 669-675.

[16] Y. Schwartzburg, M. Pauly, Fabrication-aware design with intersecting planar pieces, Computer Graphics Forum 32 (2pt3) (2013) 317-326.

[17] P. Cignoni, N. Pietroni, L. Malomo, R. Scopigno, Field-aligned mesh joinery, ACM Transactions on Graphics (TOG) 33 (1) (2014) 11.

[18] P. Song, C.-W. Fu, D. Cohen-Or, Recursive interlocking puzzles, ACM Transactions on Graphics (TOG) 31 (6) (2012) 128.

[19] P. Song, Z. Fu, L. Liu, C.-W. Fu, Printing 3d objects with interlocking parts, Computer Aided Geometric Design 35 (2015) 137-148.

[20] P. Song, B. Deng, Z. Wang, Z. Dong, W. Li, C.-W. Fu, L. Liu, Cofifab: coarse-to-fine fabrication of large $3 \mathrm{~d}$ objects, ACM Transactions on Graphics (TOG) 35 (4) (2016) 45

[21] H. Zimmer, F. Lafarge, P. Alliez, L. Kobbelt, Zometool shape approximation, Graphical Models 76 (5) (2014) 390-401.

[22] J. Vanek, J. Galicia, B. Benes, R. Měch, N. Carr, O. Stava, G. Miller, Packmerger: A 3d print volume optimizer, Computer Graphics Forum 33 (6) (2014) 322-332.

[23] J. Vanek, J. A. G. Galicia, B. Benes, Clever support: Efficient support structure generation for digital fabrication, Computer Graphics Forum 33 (5) (2014) 117-125.

[24] Y. Yang, S. Chai, X.-M. Fu, Computing interior support-free structure via hollow-to-fill construction, Computers \& Graphics

[25] C. Schumacher, B. Bickel, J. Rys, S. Marschner, C. Daraio, M. Gross, Microstructures to control elasticity in 3d printing, ACM Transactions on Graphics (TOG) 34 (4) (2015) 136.

[26] J. Panetta, Q. Zhou, L. Malomo, N. Pietroni, P. Cignoni, D. Zorin, Elastic textures for additive fabrication, ACM Transactions on Graphics (TOG) 34 (4) (2015) 135

[27] B. Zhu, M. Skouras, D. Chen, W. Matusik, Two-scale topology optimization with microstructures, ACM Transactions on Graphics (TOG) 36 (5) (2017) 164

[28] J. Panetta, A. Rahimian, D. Zorin, Worst-case stress relief for microstructures, ACM Transactions on Graphics (TOG) 36 (4) (2017) 122.

[29] C. Jiang, C. Tang, H.-P. Seidel, P. Wonka, Design and volume optimization of space structures, ACM Transactions on Graphics (TOG) 36 (4) (2017) 159.

[30] L. D. Landau, E. Lifshitz, Theory of Elasticity, Course of Theoretical Physics, Elsevier, 1986.

[31] M. Ameen, Computational Elasticity: Theory of Elasticity and Finite and Boundary Element Methods, Alpha Science Int'l Ltd., 2005.

[32] T. J. Hughes, The finite element method: linear static and dynamic finite element analysis, Courier Corporation, 2012.

[33] L. J. Gibson, M. F. Ashby, Cellular solids: structure and properties, Cambridge university press, 1999.

[34] C. L. Dym, Structural Modeling and Analysis, Cambridge University Press, 1997.

[35] J. Heyman, The Science of Structural Engineering, World Scientific, 1999.

[36] K. D. Hjelmstad, Fundamentals of Structural Mechanics, Springer Sci- ence \& Business Media, 2007.

[37] I. R. de Farias, M. Zhao, A polyhedral study of the semi-continuous knapsack problem, Mathematical Programming 142 (1-2) (2013) 169-203.

[38] X. Sun, X. Zheng, D. Li, Recent advances in mathematical programming with semi-continuous variables and cardinality constraint, Journal of the Operations Research Society of China 1 (1) (2013) 55-77.

[39] D. Bienstock, Computational study of a family of mixed-integer quadratic programming problems, Mathematical programming 74 (2) (1996) 121140

[40] G. Fung, O. Mangasarian, Equivalence of minimal $\ell_{0}$-and $\ell_{p}$-norm solutions of linear equalities, inequalities and linear programs for sufficiently small p, Journal of optimization theory and applications 151 (1) (2011) $1-10$.

[41] J. Eckstein, D. P. Bertsekas, On the douglas-rachford splitting method and the proximal point algorithm for maximal monotone operators, Mathematical Programming 55 (1) (1992) 293-318.

[42] J. Yang, Y. Li, X. Xie, Z. Yang, Alternating direction method for separable variables under pair-wise constraints, Communications in Mathematics and Statistics 5 (1) (2017) 59-82.

[43] S. Boyd, N. Parikh, E. Chu, B. Peleato, J. Eckstein, Distributed optimization and statistical learning via the alternating direction method of multipliers, Foundations and Trends $($ in Machine Learning 3 (1) (2011) $1-122$.

[44] E. Chu, A. Keshavarz, S. Boyd, A distributed algorithm for fitting generalized additive models, Optimization and Engineering 14 (2) (2013) 213 224

[45] J. Nocedal, S. J. Wright, Numerical Optimization, Springer, 2006 